\title{
Statically Typed String Sanitation Inside a Python
}

\author{
Nathan Fulton Cyrus Omar Jonathan Aldrich \\ Carnegie Mellon University \\ \{nathanfu, comar, aldrich\}@cs.cmu.edu
}

\begin{abstract}
Web applications must ultimately command systems like web browsers and database engines using strings. Strings derived from improperly sanitized user input can as a result be a vector for command injection attacks. In this paper, we introduce regular string types, which classify strings constrained statically to be in a regular language specified by a regular expression. Regular strings support standard string operations like concatenation and substitution, as well as safe coercions, so they can be used to implement, in an essentially conventional manner, the pieces of a web application or framework that handle strings arising from user input. Simple type annotations at function interfaces can be used to statically verify that sanitization has been performed correctly without introducing redundant run-time checks. We specify this type system first as a minimal typed lambda calculus, $\lambda_{R S}$.

To be practical, adopting a specialized type system like this should not require the adoption of a new programming language. Instead, we advocate for extensible type systems: new type system fragments like this should be implemented as libraries atop a mechanism that guarantees that they can be safely composed. We support this with two contributions. First, we specify a translation from $\lambda_{R S}$ to a calculus with only standard strings and regular expressions. Then, taking Python as a language with these constructs, we implement the type system together with the translation as a library using typy, an extensible static type system for Python.
\end{abstract}

Categories and Subject Descriptors F.3.3 [Logics \& Meanings of Programs]: Studies of Program Constructs-Type structure

Keywords type systems, regular expressions, input sanitation, string sanitation, extensible languages, web security

Permission to make digital or hard copies of part or all of this work for personal or classroom use is granted without fee provided that copies are not made or distributed for profit or commercial advantage and that copies bear this notice and the full citation on the first page. Copyrights for third-party components of this work must be honored.

For all other uses, contact the owner/author(s).

PSP '14, October 21, 2014, Portland, OR, USA..

Copyright is held by the owner/author(s).

ACM 978-1-4503-2296-6/14/10.

http://dx.doi.org/10.1145/2687148.2687152

\section{Introduction}

Command injection vulnerabilities are among the most common and severe security vulnerabilities in modern web applications [11]. They arise because web applications, at their boundaries, control external systems using commands represented as strings. For example, web browsers are controlled using HTML and Javascript sent from a server as a string, and database engines execute SQL queries also sent as strings. When these commands include substrings derived from user input, care must be taken to ensure that the user cannot subvert the intended command. For example, a SQL query constructed using string concatenation exposes a SQL injection vulnerability:

'SELECT * FROM users WHERE name $="$, + name + '",

If a malicious user enters the name '"; DROP TABLE users --', the entire database could be erased.

To avoid this problem, developers are cautioned to sanitize user input. For example, in this case, the developer (or, more often, a framework) might define a function sanitize that escapes double quotes and existing backslashes with a backslash, which SQL treats securely. Alternatively, it might HTML-encode special characters, which would head off both SQL injection attacks and cross-site scripting attacks. Guaranteeing that user input has already been sanitized before it is used to construct a command is challenging, especially because sanitization and command construction may occur in separately developed and maintained components.

We observe that many such sanitization techniques can be specified using regular languages [7]. For example, for the command constructed above to be secure, name must be a string in the language described by the regular expression $([\wedge " \backslash \backslash]|(\backslash ")|(\backslash \backslash)) *$ - a sequence of characters other than quotation marks and backslashes; these can only appear escaped. This conventional syntax for regular expression patterns can be understood to desugar, in a standard way, to the syntax for regular expressions shown in Figure 1, where $r \cdot r$ is sequencing and $r+r$ is disjunction. We will work with this "core" for simplicity in the remainder.

In this paper, we present a static type system that tracks the regular language a string belongs to (we identify regular languages by the notation $\mathcal{L}\{r\}$ ). For example, the output of sanitize will not simply be string, but rather 
stringin $[r]$, where $r$ is the regular expression above. By leveraging closure and decidability properties of regular languages, the type system will be able to closely track the language a string belongs to through uses of a number of operations, including replacement of substrings matching a given pattern. This makes it possible to implement sanitization functions - like the one just described - in a conventional manner. The result is a system where the fact that a string has been correctly sanitized becomes manifest in its type. Missing calls to sanitization functions can thus be detected statically, and, crucially, so can incorrectly implemented sanitization functions (i.e. these functions need not be trusted). These guarantees require run-time checks only when going from less precise to more precise types.

We will begin in Sec. 2 by specifying this type system minimally, as a conservative extension of the simply typed lambda calculus called $\lambda_{R S}$. This allows us to specify the guarantees that the type system provides precisely. We also formally specify a translation from this calculus to a typed calculus with only standard strings and regular expressions, intending it as a guide to language implementors interested in building this feature into their own languages. This also demonstrates that no additional space overhead is required.

Waiting for a language designer to build this feature in is unsatisfying in practice. We take the position that a better path forward for the community is to work within a programming language where such type system fragments can be introduced modularly and orthogonally, as libraries.

In Sec. 3, we show how to implement the type system and translation from Sec. 2 using typy, an extensible static type system implemented as a library inside Python. typy leverages local type inference to control the semantics of literal forms, so regular string types can be introduced using string literals without any run-time overhead. Coercions that are known to be safe due to a sublanguage relationship are performed implicitly, also without run-time overhead. This results in a usably secure system: working with regular strings differs little from working with standard strings in a language that web developers have already widely adopted.

We conclude after discussing related work in Sec. 4.

\section{Regular String Types, Minimally}

This section is organized as follows:

- Sec. 2.1 describes $\lambda_{R S}$ and its metatheory.

- Sec. 2.2 describes a simple target language, $\lambda_{P}$, with a minimal regular expression library. In Section 3, we will take Python to be such a language.

- Sec. 2.3 describes the translation from $\lambda_{R S}$ to $\lambda_{P}$ and ensures the correctness result from Sec. 2.1 is preserved under this translation.

The accompanying technical report [5] contains more detailed proofs and further discussion of design choices. $r::=\epsilon|\cdot| a|r \cdot r| r+r \mid r *$

$a \in \Sigma$

Figure 1. Regular expressions over the alphabet $\Sigma$.

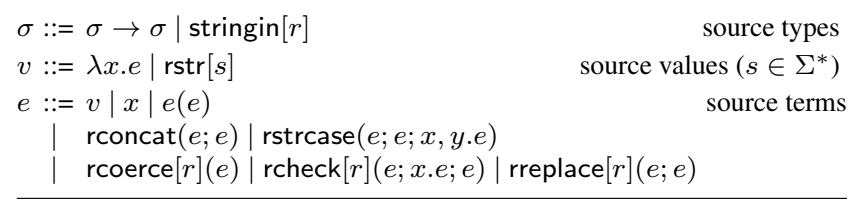

Figure 2. Syntax of $\lambda_{R S}$.

\subsection{The Language of Regular Strings}

In this section, we define a typed lambda calculus with regular string types called $\lambda_{R S}$. Its syntax is specified in Figure 2, its static semantics in Figure 3 and its evaluation semantics, given here in big-step style, in Figure 4.

There are two type constructors in $\lambda_{R S}: \rightarrow$ and stringin. Arrow types classify functions, which are introduced via lambda abstraction, $\lambda x . e$, and can be applied, written $e(e)$, in the usual way [6]. Regular string types are of the form stringin $[r]$, where $r$ is a regular expression. Values of such regular string types take the form $\operatorname{rstr}[s]$, where $s$ is a string (i.e. $s \in \Sigma^{\star}$, where the Kleene star is defined in the usual way). The rule S-T-STRINGIN-I requires that $s \in \mathcal{L}\{r\}$.

$\lambda_{R S}$ provides several familiar operations on strings. The type system relates these operations over strings to corresponding operations over the regular languages they belong to. Since these operations over regular languages are known to be closed and decidable, we can use these operations as a basis for implementing and reasoning about sanitation protocols, as we will discuss below.

\subsubsection{Concatenation}

The S-T-CONCAT rule is the simplest example of our approach. The rule is sound because the result of concatenating two strings, the first in $\mathcal{L}\left\{r_{1}\right\}$ and the second in $\mathcal{L}\left\{r_{2}\right\}$, will always be in the language $\mathcal{L}\left\{r_{1} \cdot r_{2}\right\}$. The rule therefore relates string concatenation to sequential composition of regular expressions.

\subsubsection{String Decomposition}

Whereas concatenation allows the construction of large strings from smaller strings, rstrcase $\left(e ; e_{0} ; x, y . e_{1}\right)$ allows the decomposition, or elimination, of large strings into smaller strings. Intuitively, this operation branches based on whether a string is empty or not, exactly analagous to case analysis on lists in a functional language. The branch for a non-empty string "peels off" the first character, binding it and the remainder of the string to specified variables. The evaluation rules S-E-CASE- $\epsilon$ and S-E-CASE-CONCAT express this semantics. This construct can be used to implement a standard string indexing operation as a function, given a suitable definition of natural numbers (omitted).

The typing rule S-T-CASE must determine a suitable type for the head and tail of the string. The regular expression recognizing any one-character prefix of the strings in $\mathcal{L}\{r\}$ is easily defined. 


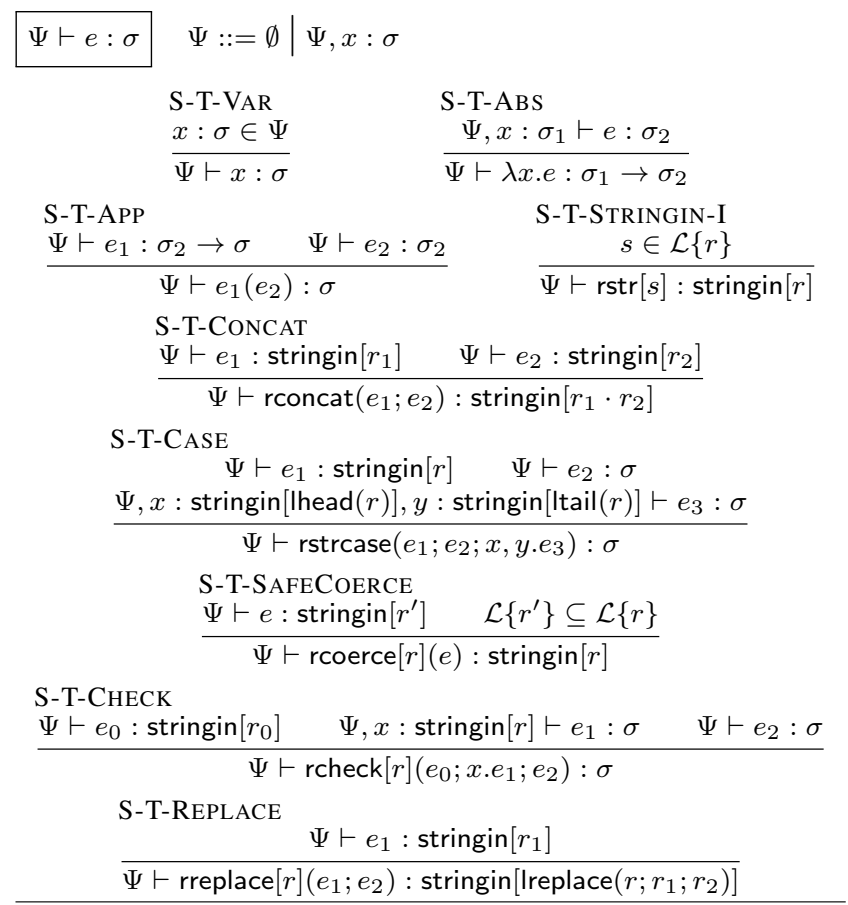

Figure 3. Typing rules for $\lambda_{R S}$. The typing context $\Psi$ is standard.

Definition 1 (Definition of lhead $(r)$ ).

$$
\operatorname{lnead}(r)=\operatorname{lnead}(r, \epsilon)
$$

We use a two-argument auxiliary $\operatorname{lhead}\left(r, r^{\prime}\right)$ in the definition of Ihead $(r)$ because when $r=q^{*} \cdot r^{\prime}, \operatorname{Ihead}(r)$ needs to "remember" $r^{\prime}$ in case $q$ is iterated zero times:

$$
\begin{aligned}
\operatorname{Ihead}\left(\epsilon, r^{\prime}\right) & =\epsilon \\
\operatorname{Ihead}\left(a, r^{\prime}\right) & =a \\
\operatorname{Ihead}\left(r_{1} \cdot r_{2}, r^{\prime}\right) & =\operatorname{lhead}\left(r_{1}, r_{2}\right) \\
\operatorname{Ihead}\left(r_{1}+r_{2}, r^{\prime}\right) & =\operatorname{lhead}\left(r_{1}, r^{\prime}\right)+\operatorname{Ihead}\left(r_{2}, r^{\prime}\right) \\
\operatorname{Ihead}\left(r^{*}, r^{\prime}\right) & =\operatorname{lhead}\left(r^{\prime}, \epsilon\right)+\operatorname{Ihead}(r, \epsilon)
\end{aligned}
$$

Given this definition of $\operatorname{lnead}(r)$, regular expression derivatives [2] provide a useful tool for defining Itail $(r)$.

Definition 2 (Brzozowski's Derivative). The derivative of $r$ with respect to $s$ is $\delta_{s}(r)=\{t \mid s t \in \mathcal{L}\{r\}\}$.

Definition 2 is equivalent to the definition given in [2], although we refer the unfamiliar reader to [12]. Definition 2 is equivalent to Definition 3.1 in both papers. We now define Itail $(r)$ using derivatives with respect to $\operatorname{lnead}(r)$.

Definition 3 (Definition of Itail $(r)$ ). Itail $(r)$ is defined in terms of Ihead $(r)$. Note that Ihead $(r)=a_{1}+a_{2}+\ldots+a_{i}$. We define Itail $(r)=\delta_{a_{1}}(r)+\delta_{a_{2}}(r)+\ldots+\delta_{a_{i}}(r)+\epsilon$.

The S-T-CASE rule, which is defined in terms of these operations, thus relates the result of "peeling off" the first character of a string to regular expression derivatives.

\subsubsection{Coercion}

The $\lambda_{R S}$ language supports two forms of coercion. Safe coercions, written rcoerce $[r](e)$, allow passage to a

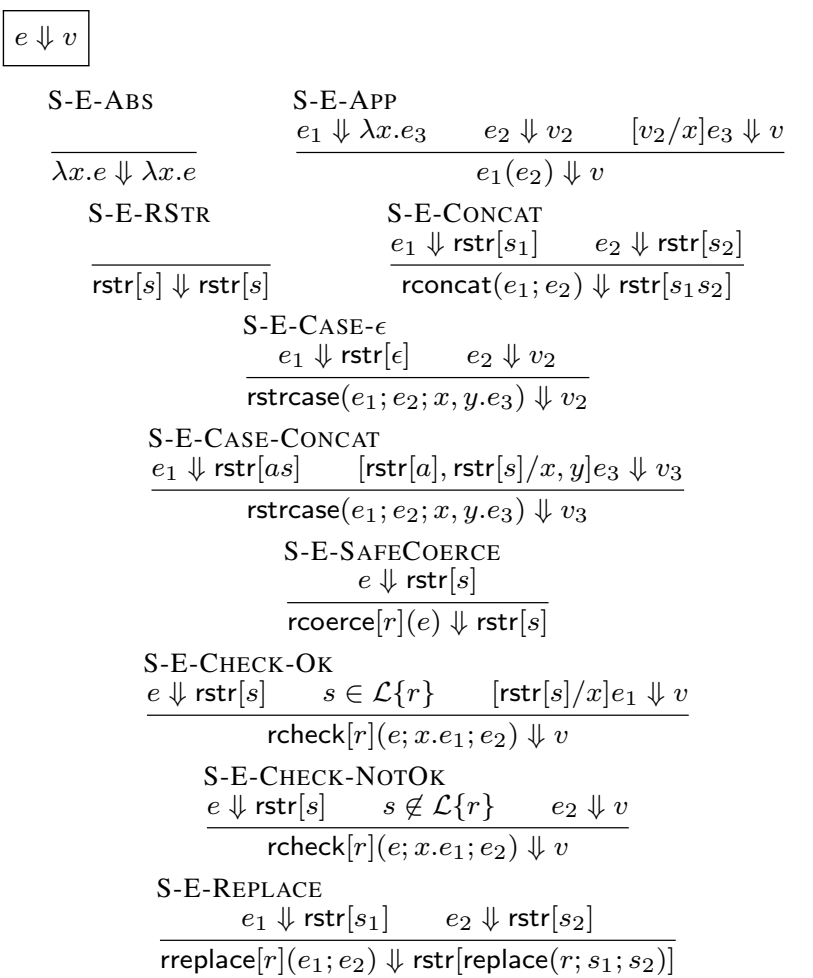

Figure 4. Big step semantics for $\lambda_{R S}$

"smaller" regular language. Such coercions will always succeed. Conversely, checked coercions, rcheck $[r]\left(e_{0} ; x . e_{1} ; e_{2}\right)$, allow for passage to regular languages that are not necessarily smaller. Checked coercions branch based on whether the coercion succeeded or not.

The rule S-T-SAFECOERCE checks for language inclusion, which we write $\mathcal{L}\left\{r_{1}\right\} \subseteq \mathcal{L}\left\{r_{2}\right\}$. Language inclusion is decidable. As a result, the rule S-E-SAFECOERCE does not need to perform any dynamic checks. The rule S-T-CHECK, conversely, does not perform any static checks on the two languages involved, only checking that the two branches have the same type. The checks are performed dynamically, by rules S-E-CHECK-OK and S-E-CHECK-NOTOK.

In our calculus, both forms of coercion are explicit. For safe coercions, it is often useful for the coercion to be performed implicitly. This can be seen as a form of subtyping for regular string types $[1,4,9]$. In practice, subtyping will be crucial to the usability of our system due to the nature of the replacement operator. For simplicity, we do not specify subtyping in our core calculus; however, subtyping for regular strings is present in previous treatments of our work [4]. The implementation discussed in Section 3 also provides subtyping between regular string types.

\subsubsection{Replacement}

The premier operation for working with regular strings in $\lambda_{R S}$ is replacement, written rreplace $[r]\left(e_{1} ; e_{2}\right)$. It behaves analogously to str_replace in PHP and string.replace in Java, differing in that the replacement pattern $r$ must be statically 
given. The evaluation rule S-E-REPLACE is defined in terms of the metafunction replace $\left(r ; s_{1} ; s_{2}\right)$.

Definition 4 (replace). replace $\left(r ; s_{1} ; s_{2}\right)=s$ such that all substrings of $s_{1}$ in $\mathcal{L}\{r\}$ are replaced with $s_{2}$.

The typing rule S-T-REPLACE thus requires computing the regular language of the string resulting from this operation given knowledge of the languages of $s_{1}$ and $s_{2}$, written as Ireplace $\left(r ; r_{1} ; r_{2}\right)=r^{\prime}$.

Given an automata-oriented interpretation of regular languages, it may be helpful to think in terms of replacing subautomata. A complete definition of Ireplace would consist of a rewrite system based on this intuition, with correctness and termination proofs, which is beyond the scope of this paper. Instead, we provide an abstract definition of the operation and state necessary properties.

Definition 5 (Ireplace). Ireplace $\left(r ; r_{1} ; r_{2}\right)$ relates $r, r_{1}$, and $r_{2}$ to a language $r^{\prime}$ containing all strings of $r_{1}$ except that any substring $s_{\text {pre }} s s_{\text {post }} \in \mathcal{L}\left\{r_{1}\right\}$ where $s \in \mathcal{L}\{r\}$ is replaced by the set of strings $s_{\text {pre }} s_{2} s_{\text {post }}$ for all $s_{2} \in \mathcal{L}\left\{r_{2}\right\}$ (the prefix and postfix positions may be empty). This procedure saturates the string.

Proposition 6 (Replacement Correspondence). Given strings $s_{1} \in \mathcal{L}\left\{r_{1}\right\}$ and $s_{2} \in \mathcal{L}\left\{r_{2}\right\}$ we have

$$
\operatorname{replace}\left(r ; s_{1} ; s_{2}\right) \in \mathcal{L}\left\{\mid \operatorname{replace}\left(r ; r_{1} ; r_{2}\right)\right\}
$$

\subsubsection{Metatheory of $\lambda_{R S}$}

In this section, we establish some basic metatheoretic properties of $\lambda_{R S}$. These rely upon the definitions and propositions given above and some basic properties of regular languages.

Lemma 7 (Properties of Regular Languages.).

1. If $s_{1} \in \mathcal{L}\left\{r_{1}\right\}$ and $s_{2} \in \mathcal{L}\left\{r_{2}\right\}$ then $s_{1} s_{2} \in \mathcal{L}\left\{r_{1} \cdot r_{2}\right\}$.

2. For all strings $s$ and regular expressions $r$, either $s \in$ $\mathcal{L}\{r\}$ or $s \notin \mathcal{L}\{r\}$.

If any of these properties are unfamiliar, the reader may refer to a standard text on the subject [7].

Type preservation for $\lambda_{R S}$ requires validating that the statics are consistent with the dynamics. We give a full proof of type safety for a variant of the calculus with a small step semantics in [5], but for concision, it is more straightforward to explain the semantics with a big step semantics here.

Theorem 8 (Type Preservation.). If $\emptyset \vdash e: \sigma$ and $e \Downarrow v$ then $\emptyset \vdash v: \sigma$.

Proof Sketch. By induction on the typing relation. The ST-CONCAT case requires Lemma 7.1, the S-T-CHECK case requires Lemma 7.2 and the S-T-Replace case appeals to Proposition 6.

We can also define a canonical forms lemma for regular strings.

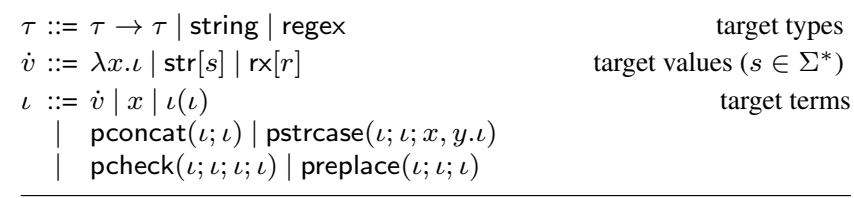

Figure 5. Syntax of the target language, $\lambda_{P}$, containing strings and statically constructed regular expressions.

Lemma 9 (Canonical Forms for Regular Strings). If $\emptyset \vdash v$ : stringin $[r]$ then $v=\operatorname{rstr}[s]$ and $s \in \mathcal{L}\{r\}$.

Proof Sketch. The only typing rule that applies is S-TSTRINGIN-I. The conclusion is the first premise.

\subsubsection{The Security Theorem}

The chief benefit of $\lambda_{R S}$ is its security theorem, which states that any term of a regular string type that evaluates to a value will evaluate to a regular string recognized by the regular language corresponding to the regular expression the type is indexed by. This is beneficial because this ensure that membership in a regular language known to be secure becomes manifest in the type of the string, rather than being a property that must be established extralinguistically.

Theorem 10 (Correctness of Input Sanitation for $\lambda_{R S}$ ). If $\emptyset \vdash e:$ stringin $[r]$ and $e \Downarrow \operatorname{rstr}[s]$ then $s \in \mathcal{L}\{r\}$.

Proof Sketch. The theorem follows directly from type preservation and canonical forms above.

\subsection{Target Language}

Our next major technical result, stated in Sec. 2.3, establishes that the security property is preserved under translation into $\lambda_{P}$. The system $\lambda_{P}$ is another straight-forward extension of a simply typed lambda calculus with a string type and a regex type, as well as some operations - such as concatenation and replacement - found in the standard libraries of many programming languages. The operations of $\lambda_{P}$ correspond to "run-time" versions of the operations performed statically in $\lambda_{R S}$ in a way made precise by the translation rules described in the next section. The language $\lambda_{P}$ is socalled because it is reminscent of popular web programming languages, such as Python or PHP, albeit statically typed. We will discuss an implementation within the former in the next section.

The grammar of $\lambda_{P}$ is defined in Figure 5. The typing rules P-T- are defined in Figure 6 and a big-step semantics is defined by the rules P-E- in Figure 7. The semantics are straightforward given the discussion of the corresponding operations in the previous section.

\subsubsection{Safety}

Type preservation for $\lambda_{P}$ is essentially trivial, but is necessary in order to establish the correctness of our translation. Again, we give a small step semantics and address type safety more completely in [5].

Theorem 11 (Safety for $\lambda_{P}$ ). If $\emptyset \vdash \iota: \tau$ and $\iota \Downarrow \dot{v}$ then $\emptyset \vdash \dot{v}: \tau$. 


$$
\begin{aligned}
& \Theta \vdash \iota: \tau \quad \Theta::=\emptyset \mid \Theta, x: \tau \\
& \text { P-T-VAR P-T-ABS } \\
& \begin{array}{l}
x: \tau \in \Theta \\
\Theta \vdash x: \tau
\end{array} \quad \frac{\Theta, x: \tau_{1} \vdash \iota_{2}: \tau_{2}}{\Theta \vdash \lambda x . \iota_{2}: \tau_{1} \rightarrow \tau_{2}} \\
& \text { P-T-APP P-T-STRING } \\
& \frac{\Theta \vdash \iota_{1}: \tau_{2} \rightarrow \tau \quad \Theta \vdash \iota_{2}: \tau_{2}}{\Theta \vdash \iota_{1}\left(\iota_{2}\right): \iota} \quad \overline{\Theta \vdash \operatorname{str}[s]: \text { string }} \\
& \text { P-T-REGEX P-T-CONCAT } \\
& \overline{\Theta \vdash r \times[r]: \text { regex }} \quad \frac{\Theta \vdash \iota_{1}: \text { string } \quad \Theta \vdash \iota_{2} \text { : string }}{\Theta \vdash \operatorname{pconcat}\left(\iota_{1} ; \iota_{2}\right): \text { string }} \\
& \text { P-T-CASE } \\
& \frac{\Theta \vdash \iota_{1}: \text { string } \quad \Theta \vdash \iota_{2}: \tau \quad \Theta, x: \text { string, } y: \text { string } \vdash \iota_{3}: \tau}{\Theta \vdash \operatorname{pstrcase}\left(\iota_{1} ; \iota_{2} ; x, y . \iota_{3}\right): \tau} \\
& \text { P-T-REPLACE } \\
& \frac{\Theta \vdash \iota_{1}: \text { regex } \quad \Theta \vdash \iota_{2}: \text { string } \quad \Theta \vdash \iota_{3}: \text { string }}{\Theta \vdash \operatorname{preplace}\left(\iota_{1} ; \iota_{2} ; \iota_{3}\right): \text { string }} \\
& \frac{\Theta \vdash \iota_{r}: \text { regex } \quad \Theta \vdash \iota_{1}: \text { string } \quad \Theta \vdash \iota_{2}: \sigma \quad \Theta \vdash \iota_{3}: \sigma}{\Theta \vdash \operatorname{pcheck}\left(\iota_{r} ; \iota_{1} ; \iota_{2} ; \iota_{3}\right): \sigma}
\end{aligned}
$$

Figure 6. Typing rules for $\lambda_{P}$. The typing context $\Theta$ is standard.

We can also define canonical forms for regular expressions and strings in the usual way:

Lemma 12 (Canonical Forms for Target Language). If $\emptyset \vdash$ $\dot{v}: \tau$ then

$$
\begin{aligned}
& \text { 1. If } \tau=\text { regex then } \dot{v}=\operatorname{rx}[r] \text { such that } r \text { is a well-formed } \\
& \text { regular expression. } \\
& \text { 2. If } \tau=\text { string then } \dot{v}=\operatorname{str}[s] \text {. }
\end{aligned}
$$

\subsection{Translation from $\lambda_{R S}$ to $\lambda_{P}$}

The translation from $\lambda_{R S}$ to $\lambda_{P}$ is defined in Figure 8. The coercion cases are most interesting. If the safety of coercion in manifest in the types of the expressions, then no runtime check is inserted (TR-SAFECOERCE). If the safety of coercion is not manifest in the type, then a check is inserted (TR-CHECK). Note that regular strings translate to strings directly; there is no space overhead.

The translation correctness theorem guarantees that the translation is type preserving and that semantics of the original code and translation coincide, following the treatment of compilation pioneered by the TIL compiler for SML [15]. Note that we apply the translation inline in judgements for concision when convenient.

Theorem 13 (Translation Correctness). If $\Theta \vdash e: \sigma$ then there exists an $\iota$ such that $\llbracket e \rrbracket=\iota$ and $\llbracket \Theta \rrbracket \vdash \iota: \llbracket \sigma \rrbracket$. Furthermore, if $e \Downarrow v$ then $\iota \Downarrow \dot{v}$ such that $\llbracket v \rrbracket=\dot{v}$.

Proof Sketch. The proof proceeds by induction on the typing relation for $e$. We choose an $\iota$ based on the syntactic form in $\lambda_{P}$ corresponding to the form under consideration (e.g. we choose replace when considering sreplace). The proof proceeds by our type safety theorems and an appeal to the induction hypothesis.

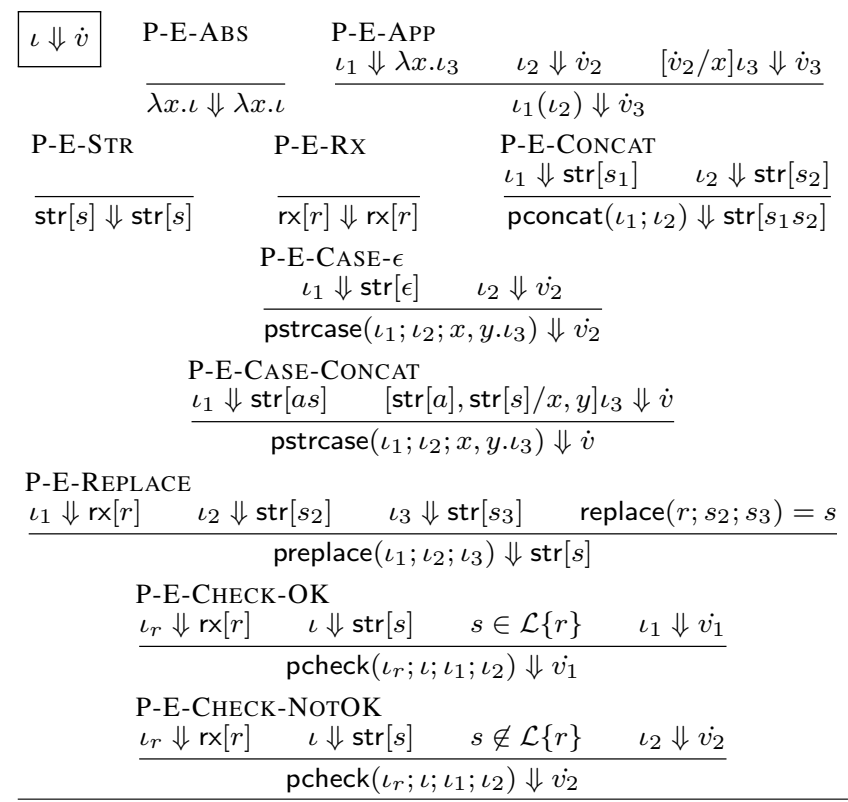

Figure 7. Big step semantics for $\lambda_{P}$

\subsubsection{Preservation of Security}

Finally, our main result establishes that correctness of $\lambda_{R S}$ is preserved under the translation into $\lambda_{P}$.

Theorem 14 (Correctness of Input Sanitation for Translated Terms). If $\llbracket e \rrbracket=\iota$ and $\emptyset \vdash e$ : stringin $[r]$ and $e \Downarrow \operatorname{rstr}[s]$ then $\iota \Downarrow \operatorname{str}[s]$ for $s \in \mathcal{L}\{r\}$.

Proof Sketch. By Theorem 13, we have our first conclusion. By Theorem 10 together with the assumption that $e$ is well-typed we have that $s \in \mathcal{L}\{r\}$.

\section{Implementation in typy}

In the previous section, we specified a type system and a translation semantics to a language containing only strings and regular expressions. In this section, we take Python to be such a target language. Python does not have a static type system, however, so to implement these semantics, we will use typy, an extensible type system for Python (being developed by the authors). By using typy, which leverages Python's quotations and reflection facilities, we can implement these semantics as a library, rather than as a new dialect of the language.

\subsection{Example Usage}

Figure 9 demonstrates the use of two type constructors, fn and stringin, corresponding to the two type constructors of $\lambda_{R S}$. We show these as being imported from typy.std, the standard library for typy (it benefits from no special support from the language itself).

The fn type constructor can be used to annotate functions that should be statically checked by typy. ${ }^{1}$ The function sanitize on lines 3-7, for example, specifies one ar-

\footnotetext{
${ }^{1}$ Here, we use argument annotation syntax only available in versions $3.0+$ of Python. Syntax supporting Python 2.7+ is available, not shown.
} 


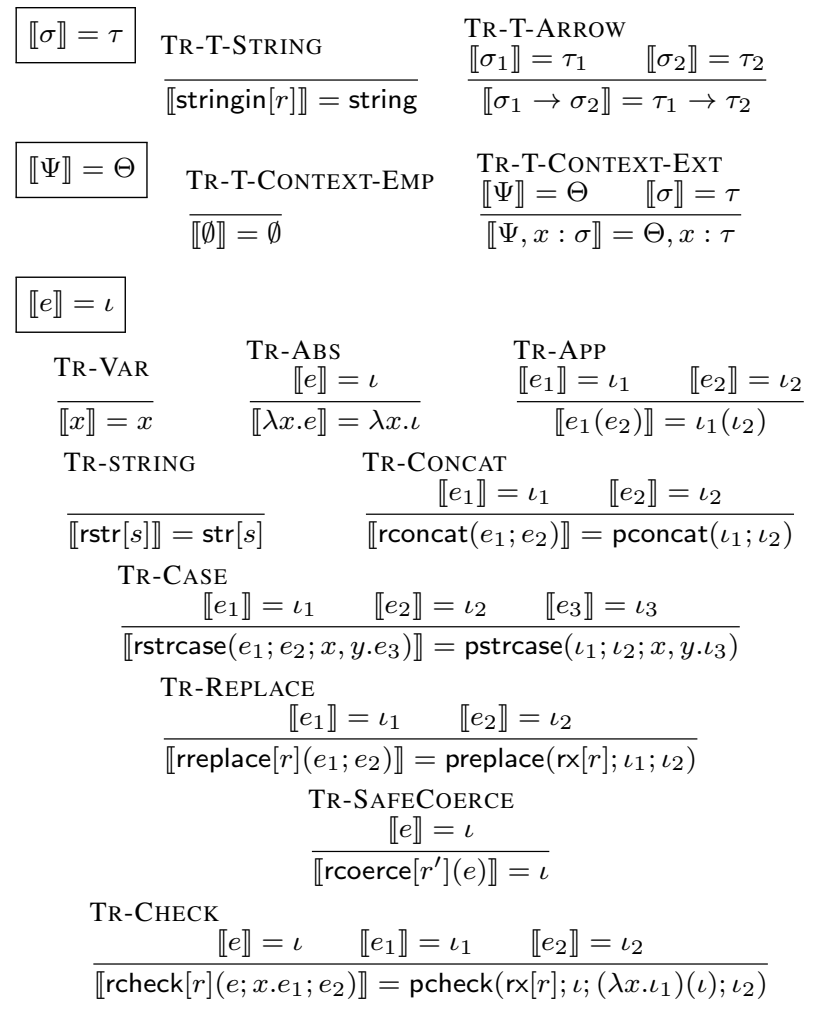

Figure 8. Translation from source to target.

gument, s, of type stringin[r'.*']. Here, the index is a regular expression, written using Python's raw string notation as is conventional (in this particular instance, the $r$ is not strictly necessary). The sanitize function takes an arbitrary string and returns a string without double quotes or left and right brackets. Note that the return type need not be specified: typy uses a form of local type inference [13].

In this example, we use an HTML encoding so that the same sanitization function can be used to generate both SQL commands and HTML securely. The sanitized string is generated by invoking the replace operator, which has the same semantics as replace in $\lambda_{R S}$. Unlike in the core calculus, it is invoked like a method on $\mathrm{s}$. The regular expression determining the substrings to be replaced is given as the first argument (as in $\lambda_{R S}$, the only restriction here is that the regular expression must be specified statically.)

The functions results_query and results_div construct a SQL query and an HTML snippet, respectively, by regular string concatenation. The argument type annotations serve as a check that sanitation was properly performed. In the case of results_query, this specification ensures that user input cannot prematurely be terminated. In the case of results_div, this specification ensures that user input does not contain any HTML tags, which is a conservative but effective policy for preventing XSS attacks. Note that the type of the surrounding string literals are determined by the type constructor of the function they appear in, $\mathrm{fn}$ in both cases, which we assume simply chooses stringin[r'.*'] (an alter-

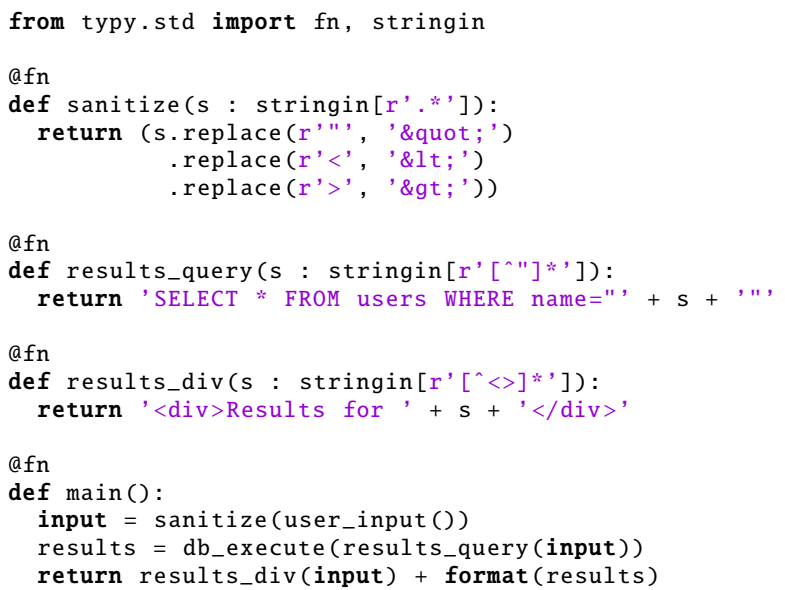

Figure 9. Regular string types in typy

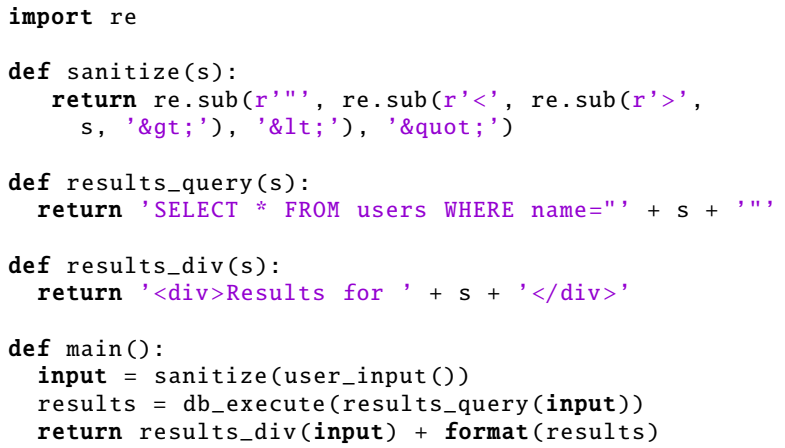

Figure 10. The output of compilation of Figure 9 (at the terminal, typy figure9.py, or just-in-time).

native strategy would be to use the most specific type for the literal, rather than the most general, but this choice is immaterial for this example). The addition operator here corresponds to the rconcat operator in $\lambda_{R S}$.

The main function invokes the functions just described. It begins by passing user input to sanitize, then executing a database query and returning HTML based on this sanitized input. The helper functions user_input and db_execute are not shown but can be assumed standard. Importantly, had we mistakenly forgotten to call sanitize, the function would not type check (in this case, it is obvious that we did, but lines 14 and 15 would in practice be separated more drastically in the code). Moreover, had sanitize itself not been implemented correctly (e.g. we forgot to strip out quotation marks), then main would also not typecheck either.

One somewhat subtle issue here is that the return type of sanitize is equivalent to stringin[ $\left[r^{\prime}\left[{ }^{\wedge} \mid<>\right]^{* \prime}\right]$, which is a distinct type from the argument types to results_query and results_div. More specifically, however, it is a "smaller" type, in that it could be coerced to these argument types using an operator like rcoerce in $\lambda_{R S}$. In our implementation, safe coercions are performed implicitly rather than explic- 


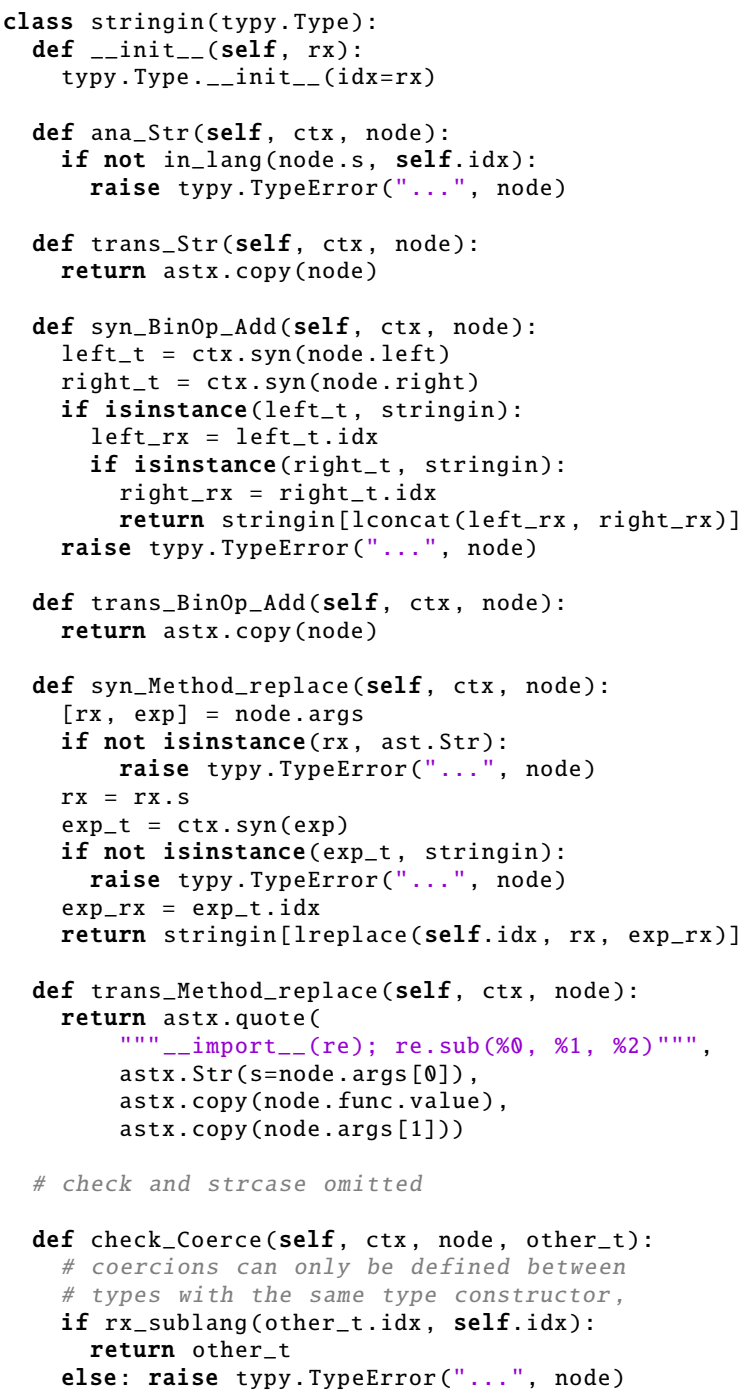

Figure 11. Implementation of stringin in typy.

itly. Because all regular strings are implemented as strings, this coercion induces no run-time change in representation.

Figure 10 shows the output of typechecking and translating this code (this can occur either in batch mode at the terminal, generating a new file, or "just-in-time" at the first callsite of each function in the dynamically typed portion of the program, not shown).

\subsection{Implementation}

The primary unit of extension in typy is the active type constructor, rather than the abstract syntax as in other work on language extensibility. This allows us to implement the entire system as a library in Python and avoid needing to develop new tooling, and also makes it more difficult to create ambiguities between extensions. Active type constructors are subclasses of typy. Type, and types are instances of these classes. The methods of active type constructors control how typechecking and translation proceed for associated operations. In Figure 11, we show key portions of the imple- mentation of the stringin type used in the example above. Although a detailed description of the extension mechanism is beyond the scope of this work, we describe the intuitions behind the various methods below.

The constructor, __init__ in Python, is called when a type is constructed. It simply stores the provided regular expression as the type index by calling the superclass.

When a string literal is being checked against a regular string type, the method ana_Str is called. It checks that the string is in the language of the regular expression provided as the type index, corresponding to rule S-T-STRINGIN-I in Section 2. The method trans_Str is called after typechecking to produce a translation. Here, we just copy the original string literal - regular strings are implemented as strings.

The method syn_Bin0p_Add synthesizes a type for the string concatenation operation if both arguments are regular strings, consistent with rule S-T-CONCAT. The corresponding method trans_Bin0p_Add again simply translates the operation directly to string concatenation, consistent with our translation semantics.

The method syn_Method_replace synthesizes a type for the "method-like" operator replace, seen used in our example. It ensures that the first argument is a statically known string, using Python's built-in ast module, and leverages an implementation of Ireplace, which computes the appropriate regular expression for the string following replacement, again consistent with our description in Section 2. Translation proceeds by using the re library built into Python, as can be seen in Figure 10.

Code for checked conversions and string decomposition is omitted, but is again consistent with our specification in the previous section. Safe coercions are controlled by the check_Coerce function, which checks for a sublanguage relationship. Here, as in the other methods, failure is indicated by raising an typy. TypeError with an appropriate error message and location.

Taken together, we see that the mechanics of extending typy with a new type constructor are fairly straightforward: we determine which syntactic forms the operators we specified in our core calculus should correspond to, then directly implement a decision procedure for type synthesis (or, in the case of literal forms, type analysis) in Python. The typy compiler invokes this logic when working with regular strings. The result is a library-based embedding of our semantics into Python.

\section{Related Work}

The input sanitation problem is well-studied. There exist a large number of techniques, proposed by both practitioners and researchers, for preventing injection attacks. In this section, we explain how our approach to the input sanitation problem differs from each of these approaches. Equally important is our assertion that language extensibility is the right approach for consideration of language-oriented security mechanisms like the one we described here. 
Unlike frameworks and libraries provided by languages such as Haskell and Ruby, our type system provides a static guarantee that input is always properly sanitized before use. Doing so requires reasoning about the operations on regular languages corresponding to standard operations on strings; we are unaware of any production system which contains this form of reasoning. Therefore, even where frameworks and libraries provide a viable interface or wrapper around input sanitation (e.g. prepared SQL statements), our approach is complementary because it can be used to ensure the correctness of that framework or library itself. Furthermore, our approach is more general than database abstraction layers because our mechanism is applicable to all forms of command injection (e.g. shell injection or remote file inclusion).

A number of research languages provide static guarantees that a program is free of input sanitation vulnerabilities by never using a string representation at all, but rather desugaring SQL syntax to a safe representation immediately [3]. The Wyvern programming language introduced a general framework for writing syntax extensions like this [10]. Unlike this work, our solution to the input sanitation problem retains a string representation and thus has a very low barrier to adoption. Our implementation conservatively extends Python - a popular language among web developers - rather than requiring the adoption of a new language entirely. We also believe our semantic extensibility approach (as opposed to only syntactic extensibility in Wyvern) is better-positioned for security, where continuously evolving threats might require frequent addition of new semantic analyses. typy is particularly well-suited to type system based analyses.

Incorporating regular expressions into the type system is not novel. The XDuce system [8] checks XML documents against schema using regular expressions. 2Similarly, XHaskell [14] focuses on XML documents. We differ from this and related work in at least three ways:

- Although our static replacement operation is definable in some languages with regular expression types, we are the first to expose this operation and connect the semantics of regular language replacement with the semantics of string replacement via a type theoretic argument.

- The underlying representation of regular strings is guaranteed to be a string, rather than a heavier implementation based on an encoding using functional datatypes.

- We demonstrate that regular expression types are applicable to the web security domain, whereas previous work on regular expression types focused on XML.

\section{Future Work}

We believe that this type system extension serves as a useful basis for web-oriented static analysis; frameworks and regular expression libraries could be annotated, along with usesites. We hope to empirically evaluate the feasability of this approach in the future using typy. We also believe that extensible programming languages are a promising approach toward incorporating other security analyses into programming languages. Construing such analyses as type systems, specifying them rigorously and implementing them within an extensible type system appears to be a promising general technique that the community may wish to emulate.

\section{Acknowledgements}

We thank the anonymous referees. This work was supported by the National Security Agency lablet contract \#H9823014-C-0140 and the National Science Foundation under NSF CNS-1035800. Any opinions, findings, and conclusions or recommendations expressed in this publication are those of the author(s) and do not necessarily reflect the views of any sponsoring institution or government.

\section{References}

[1] V. Breazu-tannen, T. Coquand, C. A. Gunter, and A. Scedrov. Inheritance as implicit coercion. Information and Computation, 93, 1991.

[2] J. A. Brzozowski. Derivatives of regular expressions. J. ACM, 11(4):481-494, Oct. 1964.

[3] A. Chlipala. Static checking of dynamically-varying security policies in database-backed applications. In OSDI'10, Oct. 2010.

[4] N. Fulton. A typed lambda calculus for input sanitation. Undergraduate thesis in mathematics, Carthage College, 2013.

[5] N. Fulton, C. Omar, and J. Aldrich. Statically typed string sanitation inside a Python. Technical Report CMU-ISR-14112, Carnegie Mellon University.

[6] R. Harper. Practical Foundations for Programming Languages. Cambridge University Press, 2012.

[7] J. E. Hopcroft and J. D. Ullman. Introduction to Automata Theory, Languages, and Computation. Addison-Wesley, 1979.

[8] H. Hosoya, J. Vouillon, and B. C. Pierce. Regular Expression Types for XML. In ICFP 'O0, 2000.

[9] K. Lu and M. Sulzmann. An implementation of subtyping among regular expression types. In W.-N. Chin, editor, Programming Languages and Systems, volume 3302 of Lecture Notes in Computer Science, pages 57-73. Springer Berlin Heidelberg, 2004.

[10] C. Omar, D. Kurilova, L. Nistor, B. Chung, A. Potanin, and J. Aldrich. Safely composable type-specific languages. In ECOOP 2014, volume 8586 of Lecture Notes in Computer Science, pages 105-130. Springer Berlin Heidelberg, 2014.

[11] OWASP. Open web application security project top 10. https://www. owasp.org/index.php/Category: OWASP_Top_Ten_Project.

[12] S. Owens, J. Reppy, and A. Turon. Regular-expression derivatives re-examined. J. Funct. Program., Mar. 2009.

[13] B. C. Pierce and D. N. Turner. Local type inference. ACM Trans. Program. Lang. Syst., 22(1):1-44, Jan. 2000.

[14] M. Sulzmann and K. Lu. XHaskell - adding regular expression types to Haskell. In Implementation and Application of Functional Languages, volume 5083 of Lecture Notes in Computer Science, pages 75-92. Springer, 2008.

[15] D. Tarditi, G. Morrisett, P. Cheng, C. Stone, R. Harper, and P. Lee. TIL: A type-directed optimizing compiler for ML. In PLDI '96, Philadelphia, PA, May 1996. 